\title{
Nonlinear resonant conversion of modes in optical waveguides
}

\author{
Federico Dios, Alexander B. Shvartsburg ${ }^{1}$, David Artigas, Ferran Canal \\ Department of Signal Theory and Communications, Universitat Politècnica de Catalunya, \\ Gran Capità s/n, Campus Nord, Edifici D.3, 08034-Barcelona, Spain
}

Received 17 October 1994; revised version received 27 April 1995

\begin{abstract}
A new class of resonant exchange of power among three co-propagating modes in dielectric optical waveguides exhibiting Kerr-like nonlinearity is reported. Unlike the coupling of modes travelling in different, spatially separated channels as in the directional coupler (Jensen, IEEE J. Quantum Electron. QE-18 (1982) 1580; Chen, Snyder and Mitchell, Electron. Lett. 26 (1990) 77; Schmidt-Hattenberger, Trutschel and Lederer, Optics Lett. 16 (1991) 294) this paper points out the possibility of obtaining an efficient coupling of modes travelling in one single channel. The key point here is the imposition of a geometrical resonance between modes, to improve the coupling process. Due to this resonance condition, which is related with the wavelength through the propagation constants, the power exchange process is very sensitive to the wavelength.
\end{abstract}

\section{Introduction}

Since the early work of Jensen [1], nonlinear exchange of power between two parallel, adjacent channels has attracted the attention of researchers due to the likelihood of obtaining power- and phase-dependent switching. Dual-core, and more recently, multiple core nonlinear couplers have been extensively investigated during recent years. The common analytic tool in this kind of device is the coupled-mode technic, using the fundamental mode of each guide o channel $[2,3]$ or, as well, the supermodes of the whole structure $[4,5]$.

In the present work we analyze the nonlinear interaction of three modes travelling along a single core guide. The goal of this work is to find the features of this modal interaction in the very specific case of considering that the differences between the propaga- tion constants of two consecutive order modes are the same, i.e. $\beta_{0}-\beta_{1}=\beta_{1}-\beta_{2}$. The underlying idea is not new: in fact we are trying to build up a periodic spatial modulation of the refractive index following the guide, and then obtaining a cumulative coupling among modes [6-8]. However, to our knowledge, this is the first complete analysis of this phenomenon in nonlinear waveguides. The net effect should be similar to that of a periodic, although nonhomogeneous grating drawn in the guide by the sum of the three modes. Unlike the periodic and homogeneous manufactured gratings that reflect the mode with the adequate wavelength, this induced grating provokes a periodic swapping of power among the modes.

In order to maintain the possibility of analyzing the phenomena with the coupled-mode theory low nonlinearity in the waveguide is assumed.

\footnotetext{
${ }^{1}$ Permanent adress: Central Design Bureau for Unique Instrumentation, Russian Academy of Sciences, Moscow P.O. 117342, Russia.
} 


\section{Analytical model}

Let us consider an slab optical waveguide composed of a core with a third-order nonlinearity, surrounded on both sides by a linear material. For simplicity we can take a symmetric guide. The refractive index in the core has a profile such that three TE modes exist and, moreover, the propagation constants of the modes satisfy the resonant condition at some wavelength

$\Delta \beta=\beta_{0}-\beta_{1}=\beta_{1}-\beta_{2}$.

This condition can be obtained with a parabolic index profile, or more simply, with a three layer core. In both cases, however, an adequate design is needed, because not any arbitrary profile satisfies the condition (1).

The field along the guide can be written as a combination of the modes, taking the $z$ axis as the propagation direction, and $x$ as the transversal axis:

$E(x, z)=\sum_{i=0}^{2} F_{i}(z) E_{i}(x) \exp \left(\mathrm{i} \beta_{i} z\right)$,

where $E_{i}(x)$ represents the transversal electric field of each mode, normalized to carry power unity, and with $F_{i}(z)$ being the complex amplitude describing the effect of the nonlinear interaction.

The nonlinear wave equation, in the slowly varying envelope approximation, can be written as

$$
\begin{aligned}
& \sum_{i=0}^{2}\left(2 \mathrm{i} \beta_{i} \frac{\mathrm{d} F_{i}(z)}{\mathrm{d} z} E_{i}(x) \exp \left(\mathrm{i} \beta_{i} z\right)\right) \\
& \quad+k_{0}^{2} \chi^{(3)}|E(x, z)|^{2} E(x, z)=0,
\end{aligned}
$$

$k_{0}=2 \pi / \lambda$ being the wavenumber and $\chi^{(3)}$ the third order nonlinear coefficient for the TE modes.

Unknown amplitudes $F_{i}(z)$ can be written as $F_{i}(z)=f_{i}(z) \exp \left(\mathrm{i} \varphi_{i}(z)\right)$, with $f_{i}(z)=\left\|F_{i}(z)\right\|$. By substituting the field (2) in the wave equation (3), several terms with different phase velocity are obtained. Applying the resonance condition the remaining combination of propagation constants modulating the phase along the $z$ axis are $\beta_{0}, \beta_{1}, \beta_{2}$ and $\beta_{0}+\Delta \beta, \beta_{0}+2 \Delta \beta, \beta_{2}-\Delta \beta, \beta_{2}-2 \Delta \beta$.

The exact method to solve the equation should take into account all these terms. However, this is not strictly necessary if we look for a solution in the case of low nonlinearity, this is again a result from the slow varying envelope approximation, because we assume that $\left|\mathrm{d} F_{i} / \mathrm{d} z\right| \ll \beta_{i} F_{i}$. Moreover we can take independently the terms affected by the same spatial phase and force them to be null. As a result of this we can get a set of three nonlinear equations, one for each mode.

Before going on, it seems necessary to extend the scope of the analysis. So far, we have assumed the condition (1) is satisfied in our waveguide. In practice one can expect that the phenomenon of power conversion among modes was very sensitive to this condition. Let us introduce a new variable to check the accuracy with which the condition of resonance is being filled. We suggest:

$\beta_{0}-\beta_{1}=\Delta \beta-\delta, \quad \beta_{1}-\beta_{2}=\Delta \beta+\delta$.

In fact, for a given guide, $\delta$ will be a function of the wavelength and will become null only at some very precise point. Thus, we extend our analysis to a certain range of wavelength around the exact resonance condition point.

Coming back to the equation describing the nonlinear phenomenon, we obtain the following set of equations:

$$
\begin{aligned}
& 4 \mathrm{i} k_{0} \eta \frac{\mathrm{d} F_{0}}{\mathrm{~d} z}+k_{0}^{2} \chi^{(3)}\left[I_{00} f_{0}^{2}+2 I_{01} f_{1}^{2}+2 I_{02} f_{2}^{2}\right] F_{0} \\
& +k_{0}^{2} \chi^{(3)} I_{0} F_{1}^{2} F_{2}^{*} \exp (2 \mathrm{i} \delta z)=0, \\
& 4 \mathrm{i} k_{0} \eta \frac{\mathrm{d} F_{1}}{\mathrm{~d} z}+k_{0}^{2} \chi^{(3)}\left[2 I_{10} f_{0}^{2}+I_{11} f_{1}^{2}+2 I_{12} f_{2}^{2}\right] F_{1} \\
& \quad+2 k_{0}^{2} \chi^{(3)} I_{0} F_{0} F_{1}^{*} F_{2} \exp (-2 \mathrm{i} \delta z)=0, \\
& 4 \mathrm{i} k_{0} \eta \frac{\mathrm{d} F_{2}}{\mathrm{~d} z}+k_{0}^{2} \chi^{(3)}\left[2 I_{20} f_{0}^{2}+2 I_{21} f_{1}^{2}+I_{22} f_{2}^{2}\right] F_{2} \\
& +k_{0}^{2} \chi^{(3)} I_{0} F_{1}^{2} F_{0}^{*} \exp (2 \mathrm{i} \delta z)=0,
\end{aligned}
$$

where the constants $I_{m n}$ are field overlap integrations:

$$
\begin{aligned}
& I_{m n}=\int_{\text {core }} E_{m}^{2}(x) E_{n}^{2}(x) \mathrm{d} x, \\
& I_{0}=\int_{\text {core }} E_{0}(x) E_{1}^{2}(x) E_{2}(x) \mathrm{d} x .
\end{aligned}
$$

As it was said above, modes are normalized to power unity, and then 


$$
\int_{-\infty}^{+\infty} E_{i}^{2}(x) d x=\frac{2 \eta k_{0}}{\beta_{i}} \quad(\mathrm{~W} / \mathrm{m}),
$$

$\eta$ being the free-space wave impedance.

A system of normalized equations can be derived from (5), taking as the normalizing length: $L=4 \eta / k_{0} \chi^{(3)} I_{0}$. The new system is now:

$$
\begin{aligned}
& \mathrm{i} \frac{\mathrm{d} F_{0}}{\mathrm{~d} \tau}+\left[u_{00} f_{0}^{2}+2 u_{01} f_{1}^{2}+2 u_{02} f_{2}^{2}\right] F_{0} \\
& \quad+F_{1}^{2} F_{2}^{*} \exp (2 \mathrm{i} \delta z)=0, \\
& \mathrm{i} \frac{\mathrm{d} F_{1}}{\mathrm{~d} \tau}+\left[2 u_{10} f_{0}^{2}+u_{11} f_{1}^{2}+2 u_{12} f_{2}^{2}\right] F_{1} \\
& \quad+2 F_{0} F_{1}^{*} F_{2} \exp (-2 \mathrm{i} \delta z)=0, \\
& \mathrm{i} \frac{\mathrm{d} F_{2}}{\mathrm{~d} \tau}+\left[2 u_{20} f_{0}^{2}+2 u_{21} f_{1}^{2}+u_{22} f_{2}^{2}\right] F_{2} \\
& \quad+F_{1}^{2} F_{0}^{*} \exp (2 \mathrm{i} \delta z)=0,
\end{aligned}
$$

where $\tau=z / L$ is the normalized propagation distance, and normalized overlap integrations, $u_{m n}=I_{m n} / I_{0}$, have been used.

If the real and imaginary parts of the system are separated $(6)$, and the power being carried by each mode $\left(W_{i}=f_{i}^{2}\right)$ is introduced, after some manipulations we obtain:

$$
\begin{aligned}
& \frac{\mathrm{d} W_{0}}{\mathrm{~d} \tau}=-2 W_{1} f_{0} f_{2} \sin \varphi, \quad \frac{\mathrm{d} W_{1}}{\mathrm{~d} \tau}=4 W_{1} f_{0} f_{2} \sin \varphi, \\
& \frac{\mathrm{d} W_{0}}{\mathrm{~d} \tau}=-2 W_{1} f_{0} f_{2} \sin \varphi,
\end{aligned}
$$

and

$$
\begin{aligned}
& \frac{d \varphi}{\mathrm{d} \tau}=\left(A W_{0}+B W_{1}+C W_{2}\right) \\
& +\frac{4 W_{0} W_{2}-W_{1}\left(W_{0}+W_{2}\right)}{f_{0} f_{2}} \cos \varphi+2 \delta L,
\end{aligned}
$$

where $\varphi(\tau)=2 \varphi_{1}-\left(\varphi_{0}+\varphi_{2}\right)$ is actually the only combination of phases of the individual modes that plays a role in the nonlinear process. The coefficients $A, B$ and $C$ are related with the overlap integrations as

$$
\begin{aligned}
& A=4 u_{10}-u_{00}-2 u_{20}, \quad B=2\left(u_{11}-u_{01}-u_{21}\right) \\
& C=4 u_{12}-2 u_{02}-u_{22} .
\end{aligned}
$$

\section{System invariants}

From direct inspection of the system (7), two invariant magnitudes are immediately deduced:

$$
\begin{aligned}
& P=W_{0}(\tau)+W_{1}(\tau)+W_{2}(\tau), \\
& \Delta W_{\mathrm{e}}=W_{0}(\tau)-W_{2}(\tau),
\end{aligned}
$$

$P$ being the total amount of power initially launched in the waveguide, and $\Delta W_{\mathrm{e}}$ the difference of power being carried by the even modes along the guide. The last invariant shows that the conversion of power occurs between the odd mode and the two even modes but not between even modes.

The invariants make possible to reduce the number of independent variables to two, namely, $\varphi$ and $W_{1}$, with four initial conditions, $\varphi(0), W_{1}(0), P$ and $\Delta W_{e}$, which describe the evolution of the system.

The Hamiltonian of the resulting system satisfies the following equations:

$$
\frac{\partial H}{\partial W_{1}}=-\frac{\mathrm{d} \varphi}{\mathrm{d} \tau}, \quad \frac{\partial H}{\partial \varphi}=\frac{\mathrm{d} W_{1}}{\mathrm{~d} \tau} .
$$

From here the Hamiltonian can be found as

$$
\begin{aligned}
H & =-W_{1} A\left(\frac{P+\Delta W_{\mathrm{e}}}{2}-\frac{W_{1}}{4}\right)-B \frac{W_{1}^{2}}{2} \\
& -C W_{1}\left(\frac{P-\Delta W_{\mathrm{e}}}{2}-\frac{W_{1}}{4}\right) \\
& -2 W_{1} \sqrt{\left(P-W_{1}\right)^{2}-\Delta W_{\mathrm{e}}^{2}} \cos \varphi-2 \delta L W_{1} .
\end{aligned}
$$

The evolution of the mode power along the guide can be obtained by solving the system given in Eqs. $(7),(8)$ with some numerical procedures, such as, the Runge-Kutta method.

Before doing this it is necessary to choose the kind of initial excitation that could give the most interesting features. As the evolution of the even modes is the same, due to the second invariant, an initial excitation with $P \gg \Delta W_{\mathrm{e}}$ should be chosen in order to obtain a maximum power exchange. On the other hand, the Hamiltonian of the system becomes phase independent if $W_{0}(0)=0$ or $W_{1}(0)=0$ is chosen. That means we can obtain a phase independent power conversion process just only by selecting one of these possibilities. Therefore, the most promising features should be expected with $W_{1}(0)+W_{i}(0)=P, W_{2-i}(0)=0$ $(i=0$ or 2$)$ and $W_{1}(0) \gg W_{i}(0)$. 


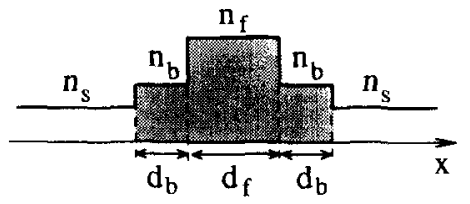

Fig. 1. The five layer slab waveguide on which numerical results are given. In an optical guide with a refractive index like that depicted here, the resonance condition among three modes $\left(\beta_{0}-\beta_{1}=\beta_{1}-\beta_{2}\right)$ can be obtained with an adequate design. The shadow core is a Kerr-like material.
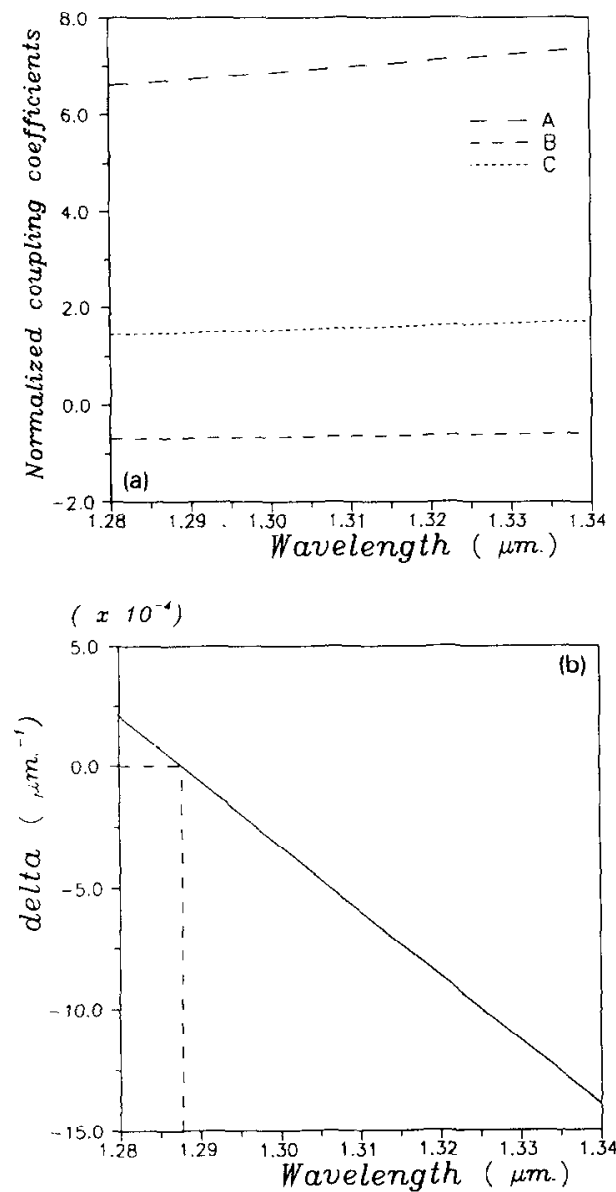

Fig. 2. (a) Normalized coupling coefficients among three modes as a function of the wavelength; (b) The delta parameter measures the proximity to the resonance condition (1) for the three modes in the waveguide.

\section{Results}

The results shown in this section have been obtained with a three-layer core slab waveguide( or five layer guide) as depicted in Fig. 1, where the nonlinearity is

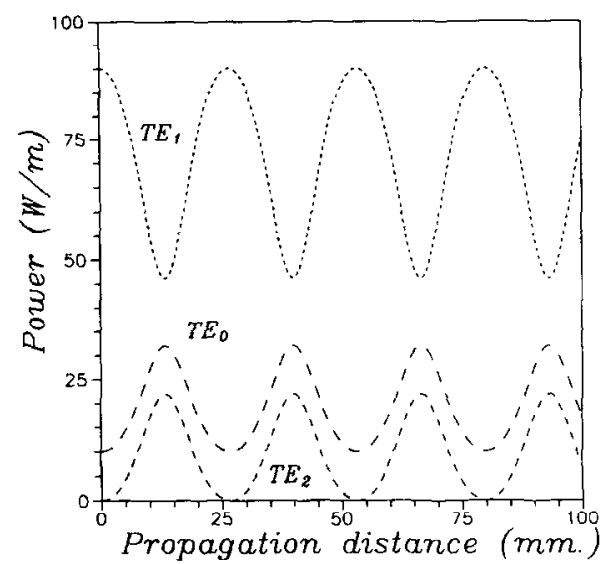

Fig. 3. Power swapping between even and odd modes, just working in the resonance wavelength. Theory shows that the even modes ( $\mathrm{TE}_{0}$ and $\mathrm{TE}_{2}$ ) act along the process as a unity, exchanging their power simultaneously with the odd mode.

located in the core, the substrate and the cover being linear media. As was stated above, the resonance condition for three modes can be obtained with this kind of guide at one particular wavelength. The values of indices and thicknesses chosen were: $n_{\mathrm{s}}=n_{\mathrm{c}}=1.451$, $n_{\mathrm{b}}=1.461, n_{f}=1.482, d_{\mathrm{b}}=2 \mu \mathrm{m}$ and $d_{f}=4 \mu \mathrm{m}$. The coefficient of nonlinearity is $\chi^{(3)}=5.795 \times 10^{-14}$ $\mathrm{m}^{2} / \mathrm{V}^{2}$ (i.e. $n_{2 f} \simeq 10^{-11} \mathrm{~m}^{2} / \mathrm{W}$ ). The plots of the Fig. 2 show: (a) the evolution of the normalized coupling coefficients $A, B$ and $C$, and (b) the shift over the resonance condition $\delta$ as a function of the wavelength. The value of the normalizing length in this case is $L \simeq 700 \mathrm{~mm}$.

In Fig. 3 the evolution of the power for each mode is shown only for $\delta=0(\lambda=1,28775 \mu \mathrm{m})$. Initial conditions can be read from plots: $W_{0}(0)=10 \mathrm{~W} / \mathrm{m}$, $W_{1}(0)=90 \mathrm{~W} / \mathrm{m}$ and $W_{2}(0)=0(\varphi(0)=0$ but this is irrelevant due to the no phase dependence in the elected excitation). The curves of mode power evolution with the propagation distance are similar in all the cases, the modulation depth and the period of the beat depending on the concrete initial conditions and the shift $\delta$. As the best features of the power process between odd modes and even modes are obtained with a particular strategy, as was explained above, we have preferred to maintain fixed the excitation and to study the evolution of the phenomenon with the wavelength. By doing this, the first effect one gets is that the maximum power exchange does not occurs at the nominal wavelength for which $\delta=0$, but for other values, be- 

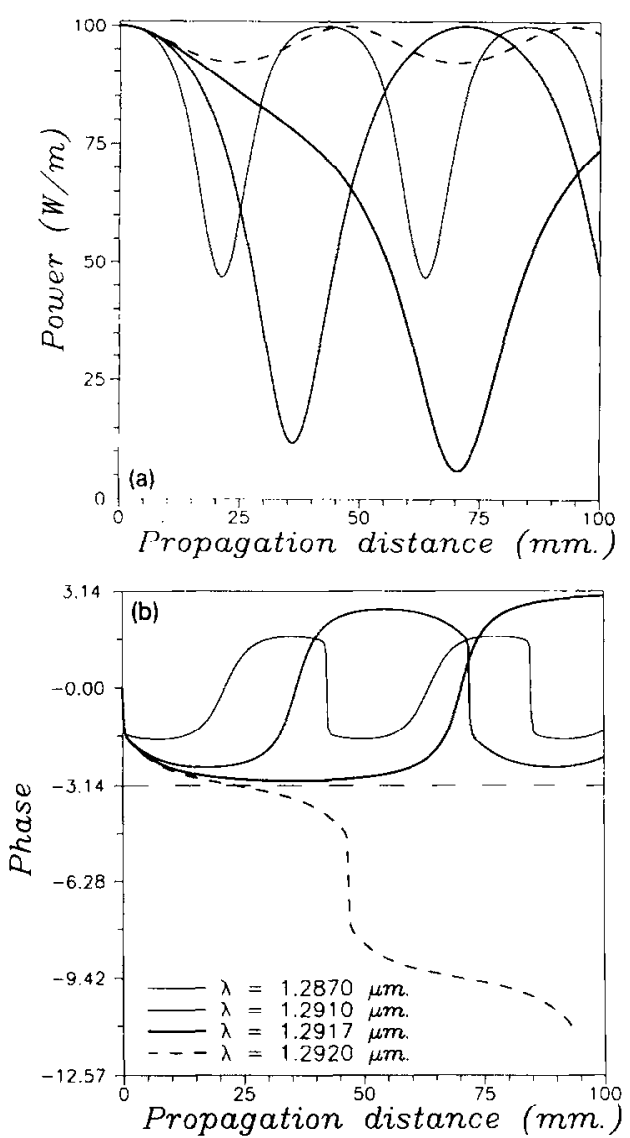

Fig. 4. Evolution of the power of the odd mode (a), and the phase shift among modes $\left(2 \varphi_{1}-\varphi_{0}-\varphi_{2}\right)$ (b), for different wavelengths. Maximum power swapping does not occur for the resonance wavelength: this is a function of the total power launched into the guide.

ing these a function of the total power launched into the guide. In other words, the real resonance condition point is not that given by linear condition (1), but one close to that, because the nonlinearity modifies slightly the effective propagation constants of the modes.

In Fig. 4a, the power evolution of the odd mode $\left(W_{1}\right)$ for different wavelengths is plotted as a func tion of the propagation distance. The corresponding phase shift is plotted in Fig. 4b. Values for $W_{0}(0)=$ $1.0 \mathrm{~W} / \mathrm{m}, W_{1}(0)=100 \mathrm{~W} / \mathrm{m}$ and $W_{2}(0)=0$ are taken. The main characteristics of the nonlinear process can be observed in these plots: first, almost all the complete exchange of power between the odd and the even modes takes place, and second, the maximum exchange occurs with a shift in wavelength in

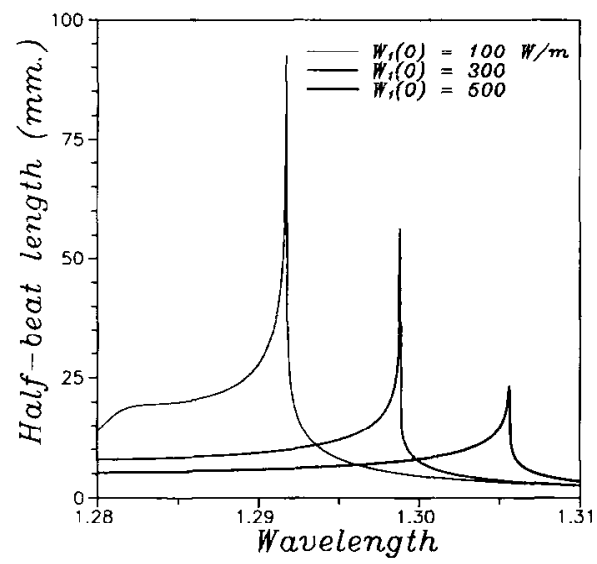

Fig. 5. Semi-period (half-beat length) of the swapping of power as a function of the wavelength, for different excitations. The peak of each plot indicates the real resonance wavelength.

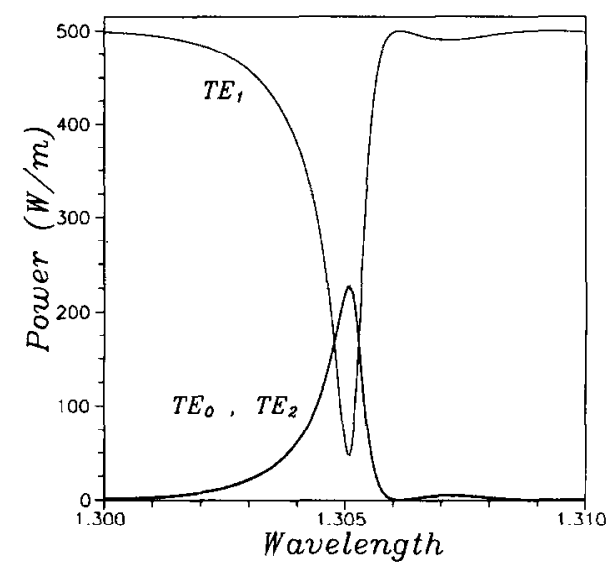

Fig. 6. The power carried by each mode at the output of the nonlinear guide for a total length of $15 \mathrm{~mm}$, as a function of the wavelength. This kind of response could be used for an optical amplifier or switch.

relation to the expected value. Finaly, it is interesting to observe that beyond the critical wavelength $(\lambda \simeq$ $1.2917 \mu \mathrm{m}$ in Fig. 4), the power exchange diminishes rapidly. With regard to the phase shift among modes ( $\varphi$ in Fig. 4b) the different behavior before and after the critical wavelength is clearly manifested.

The power of each mode has a periodic evolution in all cases. This period tends apparently to infinity as we approach that critical wavelength. In Fig. 5, this period is represented as a function of wavelength for different initial conditions: $W_{0}(0)=1.0 \mathrm{~W} / \mathrm{m}, W_{1}(0)=$ 100,300 and $500 \mathrm{~W} / \mathrm{m}$ and $W_{2}(0)=0 .(\varphi(0)$ ir- 


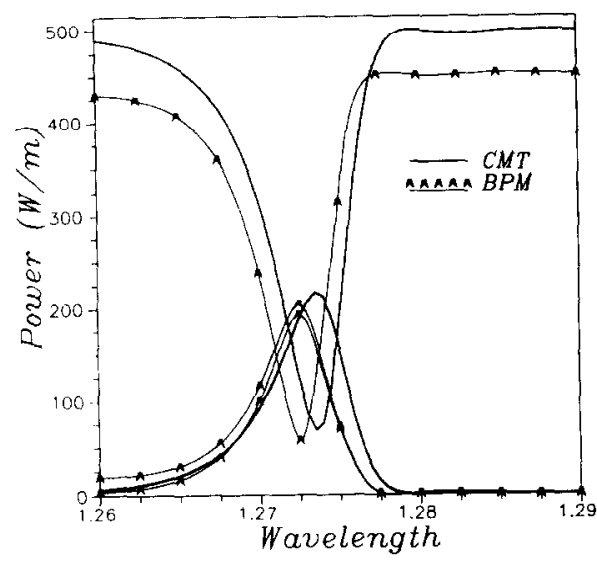

Fig. 7. Comparisons between results obtained by means of the theory reported in this work (CMT) and the well-known beam propagation method (BPM). Curves represent the mode power at the output of the nonlinear guide for a length of $8850 \mu \mathrm{m}$. Leakage of power in the BPM case must be attributed to the continuous emission of radiated modes. The comparison was made over a smoothed version of the five layer guide.

relevant.) These plots show that the resonance point in the wavelength axis can be selected by varying the total power. Actually the plain behavior of this guide would be as an optical amplifier. One can think of a signal to be amplified modulating the fundamental mode and the pumping beam in the $\mathrm{TE}_{1}$ (odd) mode. By using a guide with the appropriate length one gets the amplified signal at the output, as the even modes $\left(W_{0}+W_{2}\right)$, provided that reliable mode selectors can be designed at the input and the output. This is represented in Fig. 6. The output power for each mode for a fixed propagation distance $(z=15 \mathrm{~mm})$ is shown for a range of wavelength. At approximately $\lambda=1.305 \mu \mathrm{m}$ the initial small $\mathrm{TE}_{0}$ power is amplified $23 \mathrm{~dB}$, or 26 $\mathrm{dB}$ if the $\mathrm{TE}_{2}$ output power is also recovered.

In order to check the accuracy of the simplified coupled-mode technique (CMT) used throughout the work, we have carried out some numerical simulations by means of a FFT-beam propagation method (BPM). One of the characteristics of this method is the convenience of having a smoothed guide index profile to diminish possible numerical errors, i.e. theoreticaly abrupt transitions of the refractive index between different layers are not allowed. The practical and common solution of previously smoothing the transitions is not evident in our case: the smoothing affects the resonance condition, because it produces a slight, al- though appreciable, change in the mode propagation constants. Thus in the comparison with the CMT and the BPM we have decided to recalculate the coefficients $A, B, C, \delta$ and $L$ for the smoothed guide. Fig. 7 shows the power of each mode at the output of the guide with a total length of $8850 \mu \mathrm{m}$. A good approximation can be observed in the general behavior in the range of the wavelengths considered. Simulations were done with the initial conditions $W_{1}(0)=1.0$ $\mathrm{W} / \mathrm{m}, W_{1}(0)=500 \mathrm{~W} / \mathrm{m}$ and $W_{2}(0)=0.0 \mathrm{~W} / \mathrm{m}$.

Comparing the results obtained with both techniques two main differences appear. The first one is the total amount of power recovered at the output of the guide. This has a easy explanation, because the BPM is a step-by-step method that detects the continuous modulation of the index along the guide, and it shows that a continuous leakage of power is taking place by generation of radiated modes. The second difference is the wavelength shift of the curves. This second difference is related to the first one: i.e., the radiaton power has a secondary effect, the value of the critical wavelength (this for which the maximum power swapping occurs) being brought near the point given by the linear resonance condition. However, the accumulation of rounded-off numerical errors could also have some influence on the final result: BPM simulations were done with a transversal grid of 512 points.

Comparisons were made in the case where the nonlinear induced refractive index change is important. The highest estimated change anywhere in the waveguide was about $\Delta n \simeq 0.0018$. This shows that the proposed formalism works well enough for low nonlinearity.

Finally it is necessary to note that, as in fact a grating is induced, the bandwidth in wavelength for which an appreciable coupling occurs could be designed at will, by selecting properly the type of guide, (i.e., the slope of $\delta$ as a function of the wavelength), the total power and the length of the nonlinear guide.

\section{Conclusions}

A nonlinear induced grating can be set up in a Kerr like optical waveguide by using the periodic interference of three modes. Working near the point in the wavelength axis where $\beta_{0}-\beta_{1}=\beta_{1}-\beta_{2}$ an impor- 
tant amount of power is swept between odd and even modes. The peak of resonance location is a function of the total power into the guide. Another interesting feature of this nonlinear phenomenon is the phaseindependent behavior. The net effect with the appropriate initial excitation suggest that this kind of phenomenon could be used as the basis of optical amplifiers or switches. However, the feasibility of such a device is limited in practice by the necessity of arranging precise mode selectors at the extreme of the guide [9].

\section{Acknowledgements}

One of the authors, A. Shvartsburg, thanks the Department of Signal Theory and Communications at the Universitat Politècnica de Catalunya for its hospitality during his stay as visiting professor.

\section{References}

[1] S.M. Jensen, IEEE J. Quantum Electron. QE-18 (1982) 1580.

[2] Y. Chen, A.W. Snyder and D.J. Mitchell, Electron. Lett. 26 (1990) 77.

[3] C. Schmidt-Hattenberger, U. Trutschel and F. Lederer, Optics Lett. 16 (1991) 294

[4] Y. Silberberg and G.I. Stegeman, Appl. Phys. Lett. 50 (1987) 81.

[5] D. Artigas and F. Dios, IEEE J.Quantun Electron. 30 (1994) 1587.

[6] R.C. Youngquist, J.L. Brooks and H.J. Shaw, Optics Lett. 9 (1984) 177.

[7] B.Y. Kim, H.G. Park and H.J. Shaw, Dynamical couplers using two-mode optical waveguides, U.S. Patent no. 4.895.421. (1990).

[8] A.B. Shvartsburg, Non-linear pulses in integrated and waveguide optics (Oxford University Press, 1993).

[9] S. Berdagué and P. Facq. Appl. Optics 21 (1982) 1950 